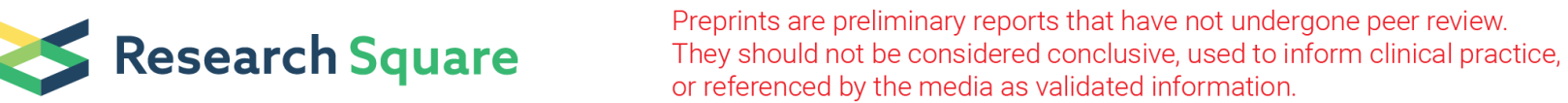

\section{Comparison of bedside index for severity in acute pancreatitis(BISAP) and modified CT severity index(MCTSI) for predicting the severity of hyperlipidemic acute pancreatitis(HLAP)}

\section{Lan Nie}

Wuhan Univesity of Science and Technoligy School of Medicine https://orcid.org/0000-0002-80091867

Yi Cheng

Chinese PLA General Hospital of Central Theater Command

\section{Fei Yao}

Wuhan University of Science and Technology school of Medicine

Ru Shuo Wu

Wuhan University of Science and Technology of Medicine

\section{Ming Li}

Chinese PLA General Hospital of Central Theater Command

\section{Junjun Zhang}

Chinese PLA General Hospital of Central Theater Command

Ming qing Wu ( $\nabla$ wuhe9224@sina.com )

Hui Long

Tianyou Hospital Affiliated to Wuhan University of Science and Technology

\section{Ming Xiang Fang}

Puren Hospital Affiliated to Wuhan University of Science and Technology

\section{Research article}

Keywords: Hyperlipidemic acute pancreatitis,Triglycerides, BISAP score,MCTSI score, Severe acute pancreatitis

Posted Date: November 15th, 2019

DOl: https://doi.org/10.21203/rs.2.17351/v1

License: () (1) This work is licensed under a Creative Commons Attribution 4.0 International License. Read Full License 


\section{Abstract}

Background: In recent years,the incidence of hyperlipidemic acute pancreatitis(HLAP) is rapidly increasing.It is important for Clinical worker to identify the severity at early stage of HLAP. AIMS: The goal of this paper was to compare bedside index for severity in acute pancreatitis(BISAP) and modified CT severity index(MCTSI) for predicting the severity and local complications of HLAP.

Methods: We collected 167 patients with HLAP, including 133 cases of Mild acute pancreatitis(MAP), 34 cases of Moderately severe acute pancreatitis(MSAP) and Severe acute pancreatitis(SAP). The study retrospectively analyzed the clinical characteristics of two groups(MAP group,MSAP and SAP group) of patients.Correlation analysis was demonstrated by Spearman,s test. In addition,the accuracy was investigated through the study of the receiver operating characteristic(ROC) curve to predict the severity of HLAP by BISAP and MCTSI score.

Results:There are significantly statistical differences $(\mathrm{P}<0.05)$ in Triglycerides $(\mathrm{TG})$, Total cholesterols(TC),Hospitalization days,Fatty liver,and Local complications between two groups,While no statistical differences in Gender, Age,Serum amylase,Alanine aminotransferase(ALT),Aspertate aminotransferase(AST),Hypertension,Type2 diabetes, and Hyperuricemia. Positive relevance was established between BISAP and TG with $r$ of 0.55 . The Area Under the Curve(AUC) of BISAP and MCTSI in predicting the severity of HLAP respectively were $0.89 \varangle 0.78 \llbracket$ sensitivity were $73.5 \% \varangle 79.4 \%$, specificity were95.5\% $₫ 60.2 \%$, positive predictive value(PPV) were $80.6 \% \bigotimes 33.8 \%$, negative predictive value(NPV) were $93.4 \% \bigotimes 92.0 \%$.In predictionig local complications,the AUC respectively were $0.73 \llbracket 0.87$, sensitivity were $37.5 \% \varangle 90.1 \%$, specificity were $93.2 \% \bigotimes 78.6 \%$, PPV were $77.4 \% \bigotimes 72.5 \%$, NPV were $70.6 \% \varangle 93.1 \%$.

Conclusion: BISAP score has a meaningful prognostic value for predicting the severity of HLAP and higher AUC than MCTSI.In particular,there are significant association between BISAP and TG.Keywords:Hyperlipidemic acute pancreatitis囚Triglycerides;BISAP score区MCTSI score; Severe acute pancreatitis

\section{Background}

Acute Pancreatitis(AP) is an acute inflammatory disease triggered by a variety of causes.According to 2012 revised Atlanta classification criteria,AP was grouped into Mild acute pancreatitis(MAP) $\square$ Moderately severe acute pancreatitis(MSAP) and Severe acute pancreatitis(SAP), with a total mortality rate of $5 \%$ $10 \%$, but the mortality rate of SAP was relatively higher,which is about $36 \% \sim 50 \%\left[{ }^{[1]}\right.$. In China, it is well established that biliary diseases are always the primary causes of AP.However, with the improvement of economic and changes in lifestyle, the incidence of hyperlipidemic acute pancreatitis(HLAP) is rapidly increasing year by year, accounting for around $10.36 \%$.Morever,there are much higher recurrences and hospitalization $\operatorname{costs}^{[2]}$.

Currently,the majority of scoring systems are applied to assess the severity of AP, including Ranson's score, the Acute Physiology and Chronic Health Evaluation (APACHE II) score, BISAP score, MCTSI score, 
et al ${ }^{[3-6]}$, but there is no unified standard clinically.Modified CT severity index(MCTSI) is a scoring system formed on the basis of CT severity index(CTSI), which could not only reflect pancreatic inflammation and necrosis, but also indicate organ failure and extrapancreatic complications ${ }^{[3]}$. Bedside index for severity in acute pancreatitis(BISAP) score was formally proposed by $\mathrm{Wu}^{[4]}$ in 2008 , the latest among other scores. When BISAP $\geq 3$, expected to be MSAP and SAP, the mortality rate markedly increased. Due to BISAP and MCTSI scores are able to repeatedly evaluate diseases condition and easy to obtain, there are utilized by more and more clinical workers.

The aims of our study were to primarily investigate the value of BISAP for predicting the severity of HLAP and compared with MCTSI.

\section{Methods}

\subsection{Data collection}

We collected 167 patients with HLAP in The General Hospital of People's Liberation Army, Tianyou Hospital Affiliated to Wuhan University of Science and Technology and Puren Hospital Affiliated to Wuhan University of Science and Technology from January 2017 to December 2018. The exclusion criterias included patients with malignant tumour, pregnancy and transferred from other hospital.Two patients were absent from further analysis for imaging information missing.

The diagnosis of AP demands at least two of the following three characteristics: \abdominal pain consistent with acute pancreatitis(acute onset of a persistent, severe, epigastric pain often radiating to the back); 『serum lipase activity (or amylase activity) at least three times greater than the upper limit of normal; and \characteristic findings of acute pancreatitis on computed tomography (CT) and less commonly magnetic resonance imaging (MRI) or transabdominal ultrasonography. In addition to above features,HLAP was diagnosed if TG met $11.0 \mathrm{mmol} / \mathrm{L}$, or ranged from 5.56 to $11.0 \mathrm{mmol} / \mathrm{L}$ but accompanied with chylemia,except for other etiologies of AP,namely,gallstone drug $\square$ infection $\square$ et al.

The category of severity(MAP $\square$ MSAP $\square$ SAP) and local compications(acute peripancreatic fluid collection, pancreatic pseudocyst, acute necrotic collection and walled-off necrosis) were in accordance with 2012 revised Atlanta criteria.

BISAP score contained ${ }^{[4]}: \bigotimes$ blood urea nitrogen $(B U N)>25 \mathrm{mg} / \mathrm{dl}$; \impaired mental status; $₫$ systemic inflammatory response syndrome(SIRS); 『age $>60$ years; 『or the presence of a pleural effusion,which were calculated within the first $24 \mathrm{~h}$ after admission(each one represents 1 point).

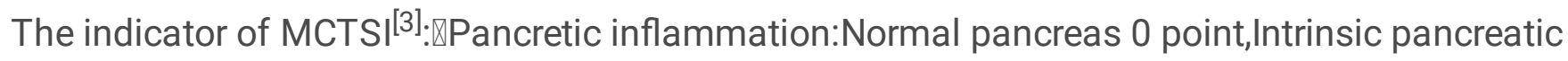
abnormalities with or without inflammatory changes in peripancreatic fat 2 points,Pancreatic or

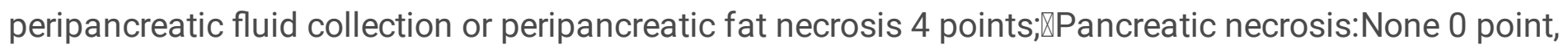

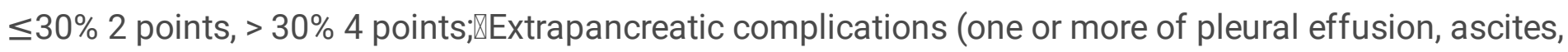


vascular complications, parenchymal complications, or gastrointestinal tract involvement) 2 points, were collected within one week of onset of symptoms and evaluated by two radiologists and one Clinical worker.

\subsection{Statistical Analysis}

SPSS24.0 software was used for statistical analysis. Descriptive data were performed as mean and standard deviation,Categorical data as frequencies and percentages.Groups comparison used Student's ttest for Descriptive data and Chi-square or Fishers' exact test for Categorical data. Correlation analysis was demonstrated by Spearman's test.Finally, ROC curve was drawn corresponding AUC and sensitivity, specificity, PPV, NPV, Youden index computed.P $<0.05$ was considered statistically significant.

\section{Results}

3.1 Comparison of clinical features between groups

As showing in table 1,table 2, table 3.The study enrolled 167 patients with HLAP.There were 133 MAP petients,with 90 male $(67.7 \%)$ and an average age of $(39.8 \pm 11.0)$ years,34 MSAP and SAP petients,with 24 male $(70.6 \%)$ and an average age of $(39.7 \pm 11.0)$ years, indicating no significant difference $(P>$ 0.05).TG, TC, hospital days and BISAP scores for MSAP and SAP patients were great higher than MAP petients $(P<0.05)$. However, ALT, AST, blood amylase had no obvious difference among groups $(P>$ 0.05).Moreover, The presence of fatty liver, local complications was higher in MSAP and SAP compared to in MAP $(P<0.05)$. The occurrence of type 2 diabetes, hypertension, hyperuricemia demonstrated no difference between the two groups $(P>0.05)$. There were statistically significant for BISAP and MCTSI scores in predicting severity and local complications $(P<0.05)$.

3.2 Comparision between BISAP and MCTSI in predicting severity and local complications of HLAP

As seen in table 4,Fig 1,Fig 2.When BISAP score $\geq 3$, in forecasting MSAP and SAP,the AUC was 0.89, sensitivity, specificity, PPV, NPV were $73.5 \% \varangle 95.5 \% \varangle 80.6 \% \varangle 93.4 \%$ respectively,Youden index was 0.69 . In forecasting local complications, the AUC was 0.73 , sensitivity, specificity, PPV, NPV were $37.5 \% \varangle 93.2 \%$ ه $77.4 \% \square 70.6 \%$ respectively,Youden index was 0.31 . When MCTSI score $\geq 4$, the AUC口Youden index $\square$ sensitivity $\square$ specificity $\square P P \square N P V$ were $0.78 \llbracket 0.87,0.40 \otimes 0.69,79.4 \% \varangle 90.1 \%$ for severity and $60.2 \% \varangle 78.6 \%$, $33.8 \% \bigotimes 72.5 \%, 92.0 \% \bigotimes 93.1 \%$ for local complications.

3.3 Correlation between BISAP and TG of HLAP

As seen in Fig 3. There are significant association between BISAP and TG with r of 0.55 .

\section{Discussion}


In 1952, Klatskin ${ }^{[7]}$ first proposed that hyperlipidemia was able to cause AP.It was widely recognized that gallstones and alcoholism were the two major contributing factors for AP, while hyperlipidemia was less frequency, only making up $4-10 \%[8]$.In recent years,people's lifestyle and dietary habits gradually westnized, which bringing about the elevated incidence for HLAP. Zheng et a ${ }^{[2]}$ investigated the changing trend of AP etiology during the period of five years in Beijing, founding that the occurrence of HLAP had been increasing year after year.In this report,HLAP surpassed alcoholic pancreatitis as the second largest etiological diagnosis.Compared to other types AP, HLAP had more complex pathological mechanism, faster aggravation, easier relapse, and the mortality rate of which was as high as $30 \%{ }^{[9]}$. Therefore,it is crucial to predict the severity at early stage for better individualized treatment and management.

Our study highlighted that there were more man(67.7\% in MAP,70.6\% in MSAP and SAP) than woman(32.3\% in MAP,29.4\% in MSAP and SAP) patients with young ages(39.8 \pm 11.0 in MAP,39.7 \pm 11.0 in MSAP and SAP ) of HLAP, which was in line with the report of Li et al ${ }^{[10]}$. This phenomenon probably was responsible for strong work and life pressure, alcohol abuse, smoking, absent exercise, high-calorie food(such as high-fat diet, high-sugar diet), and irregular sleeping schedule in this group.Additiongly, TG in MSAP and SAP group $(23.3 \pm 12.0) \mathrm{mmol} / \mathrm{L}$ was significantly higher than MAP group $(13.1 \pm 8.2)$ $\mathrm{mmol} / \mathrm{L}$.Oh et al ${ }^{[11]}$ and Yadav et al ${ }^{[12]}$ believed that the occurrence and mortality rate of AP will be reduced when TG is controlled under $5.65 \mathrm{mmom} / \mathrm{L}$.In our study,We also found that the proportion of fatty liver in MSAP and SAP group was $82.4 \%$ and $50.4 \%$ in MAP group.In accordance, a report showed that $100 \%$ patients had fatty liver in MSAP and SAP and 59\% in MAP. Besides, the severe degree of fatty liver elevated accompanied with TG increased ${ }^{[13]}$. Hence we guess that the influence of fatty liver for HLAP depends on the change of TG level.

BISAP was derived on data collected from 17992 cases of AP from 212 hospitals in 2000-2001 and validated by 18256 AP cases from 177 hospitals in 2004-2005. In this study,Wu et al concluded BISAP was as effective as APACHE II to evaluate in-hospital mortality ${ }^{[4]}$.A report demonstrated the accuracy of BISAP for severity of AP were not worse than "traditional" scoring systems(Ranson score,APACHE II,MCTSI et al $)^{[14]}$.Qiu et al ${ }^{[15]}$ observed the AUC, sensitivity, specificity respectively were $0.905,1.000,0.604$ for BISAP score in the prediction of SAP, which higher than MCTSI score with the AUC,sensitivity, specificity being $0.834,0.800,0.868$.In our study, BISAP score also have higher accuracy in predicting MSAP and SAP than MCTSI score.Moreover, we also found that BISAP had a close correlation with TG, which both were in connection with severity of HLAP.We could further hypothesize that BISAP had a high accuracy to estimate the severity risk of HLAP.

MCTSI is a major clinical imaging scoring system showed strongly correlation with the coccurrence of mortality and morbidity ${ }^{[16-17]}$.A study revealed that MCTSI manifested certain accuracy for the death of HLAP patients compared with nonhyperlipidemic acute pancreatitis(NHLAP ${ }^{[15]}$. According to the retrospective research from Yang et al[ ${ }^{[18]}$, MCTSI score had outstanding performance in the prediction of local complications but poor in severity compared to BISAP.We also found MCTSI score(AUC 0.87) had higher accuracy than BISAP score(AUC 0.73) in predicting local complications. However, there are still 
some limitations in clinical practise for MCTSI score.Firstly, We need to supervise the state repeatedly because of instantly changing of disease, which evidently lifting the economic burden of patients with contrast-enhanced CT. In addition, contrast agents are harmful to the kidney, so contrast-enhanced CT for patients with renal injury will be limited.

\section{Conclusions}

We possibly concluded that TG had a tightly relevant to severity of HLAP. BISAP score had the advantages of few parameters and easy to implement with higher AUC in the prediction of severity than MCTSI score. We are responsible to compare BISAP score with other else scoring system or integrate other scoring system and laboratory indexes to comprehensively verify severity.

\section{Abbreviations}

BISAP:bedside index for severity in acute pancreatitis;MCTSI:modified CT severity index;HLAP:hyperlipidemic acute pancreatitis;MAP:Mild acute pancreatitis;MSAP:Moderately severe acute pancreatitis;SAP:Severe acute pancreatitis;ROC:receiver operating characteristic;TG:Triglycerides;TC:Total cholesterols;AST:Aspertate aminotransferase;ALT:Alanine aminotransferase;AUC:Area Under the Curve;PPV:positive predictive value;NPV:negative predictive value;APACHE II:the Acute Physiology and Chronic Health Evaluation;CTSI:CT severity index;CT:computed tomography;MRI:magnetic resonance imaging;BUN:blood urea nitrogen;SIRS:systemic inflammatory response syndrome;NHLAP:nonhyperlipidemic acute pancreatitis.

\section{Declarations}

\section{Acknowledgements}

Not applicable

\section{Author's contribution}

L N conceived the study; L N and QM W participated in the design and drafted the manuscript;L N, SR W, F $\mathrm{Y}, \mathrm{XM} F, \mathrm{HL}$ and $\mathrm{Y} \mathrm{C}$ collected the data and performed statistical analyses.QM W, Y C, M L and JJ Z edited and checked the manuscript.All of the authors have read and approved the final manuscript.

\section{Funding}

Not applicable.

\section{Availability of data and materials}


All data generated or analysed during this study are included in this published article [and its supplementary information files]

\section{Ethics approval and consent to participate}

The study was approved by the ethics committees of the General Hospital of People's Liberation Army,Tianyou Hospital Affiliated to Wuhan University of Science and Technology and Puren Hospital Affiliated toWuhan University of Science and Technology.Written informed consent of each patient was waived as it was a retrospective study.

\section{Consent for publication}

Not applicable

\section{Competing interests}

The authors declare that they have no competing interests

\section{Tables}

Table 1 Comparison of clinical features between two groups 


\begin{tabular}{|c|c|c|c|}
\hline Features & $\begin{array}{l}\text { MAP } \\
n=133\end{array}$ & $\begin{array}{c}\text { MSAP and SAP } \\
n=34\end{array}$ & $\mathrm{P}$ value \\
\hline Gender,\% & $90(67.7)$ & $24(70.6)$ & 0.74 \\
\hline Male & $43(32.3)$ & $10(29.4)$ & \\
\hline $\begin{array}{c}\text { Female } \\
\text { Age,years } \\
\text { ALT(U/L) } \\
\text { AST(U/L) } \\
\text { TG(mmol/L) } \\
\text { TC(mmol/L) } \\
\text { Amylase(U/L) } \\
\text { BISAP }\end{array}$ & $\begin{array}{c}39.8 \pm 11.0 \\
29.1 \pm 20.0 \\
27.0 \pm 20.1 \\
13.1 \pm 8.2 \\
8.5 \pm 4.0 \\
304.7 \pm 336.7 \\
0.9 \pm 0.8\end{array}$ & $\begin{array}{c}39.7 \pm 11.0 \\
27.0 \pm 20.1 \\
32.1 \pm 31.8 \\
23.3 \pm 11.9 \\
12.0 \pm 8.9 \\
349.2 \pm 306.2 \\
2.5 \pm 0.9\end{array}$ & $\begin{array}{l}0.95 \\
0.59 \\
0.49 \\
0.00 \\
0.03 \\
0.46 \\
0.00\end{array}$ \\
\hline MCTSI & $2.9 \pm 1.4$ & $4.8 \pm 1.8$ & 0.00 \\
\hline $\begin{array}{l}\text { Hospitalization days } \\
\text { Local complication,\% }\end{array}$ & $8.3 \pm 2.7$ & $13.0 \pm 4.8$ & 0.00 \\
\hline 0 & $94(70.7)$ & $9(26.5)$ & 0.00 \\
\hline 1 & $39(29.3)$ & $25(73.5)$ & \\
\hline \multicolumn{4}{|l|}{ Hypertension,\% } \\
\hline 0 & $105(78.9)$ & $24(70.6)$ & 0.30 \\
\hline $\begin{array}{c}1 \\
\text { Hyperuricemia,\% }\end{array}$ & $28(21.1)$ & $10(29.4)$ & \\
\hline 0 & & & 0.25 \\
\hline 1 & 106(79.7) & $30(88 . .2)$ & \\
\hline Type 2 diabete,\% & $27(20.3)$ & $4(11.8)$ & 0.69 \\
\hline 0 & $87(65.4)$ & $21(61.8)$ & \\
\hline $\begin{array}{c}1 \\
\text { Fatty liver,\% }\end{array}$ & $46(34.6)$ & Fatty liver,\% & \\
\hline 0 & $66(49.6)$ & $6(17.6)$ & 0.01 \\
\hline 1 & $67(50.4)$ & $28(82.4)$ & \\
\hline
\end{tabular}

Alanine aminotransferase ALT,Aspertate aminotransferase AST,Triglycerides TG,Total cholesterols TC,Bedside index for severity in acute pancreatitis BISAP,Modified CT severity index MCTSI,Mild acute pancreatitis MAP,Moderately severe acute pancreatitis MSAP, Severe acute pancreatitis SAP, $0=$ no of cases, $1=$ yes of cases 
Table2 Analysis between BISAP and MCTSI in predicting severity of HLAP

\begin{tabular}{cccc}
\hline Scoring system & MAP & MSAP and SAP & P value \\
& $\mathrm{n}=133$ & $\mathrm{n}=34$ \\
\hline BISAP & & & \\
$\geq 3$ & $6(4.5 \%)$ & $25(73.5 \%)$ & 0.00 \\
$<3$ & $127(95.5 \%)$ & $9(26.5 \%)$ & \\
MCTSI & & & 0.00 \\
$\geq 4$ & $53(39.8 \%)$ & $27(79.4 \%)$ & $0.60)$ \\
$<4$ & $80(60.2 \%)$ & $7(20.6 \%)$ & \\
\hline
\end{tabular}

Bedside index for severity in acute pancreatitis BISAP,Modified CT severity index MCTSI,Hyperlipidemic acute pancreatitis HLAP,Mild acute pancreatitis MAP,Moderately severe acute pancreatitis MSAP, Severe acute pancreatitis SAP

Table3 Analysis between BISAP and MCTSI in predicting local complication of HLAP

\begin{tabular}{cccc}
\hline Scoring system & No Local complication & Local complication & P value \\
& $\mathrm{n}=103$ & $\mathrm{n}=64$ & \\
\hline BISAP & & & \\
$\geq 3$ & $7(6.8 \%)$ & $24(37.5 \%)$ & 0.00 \\
$<3$ & $96(93.2 \%)$ & $40(62.5 \%)$ & \\
MCTSI & & & 0.00 \\
$\geq 4$ & $22(21.4 \%)$ & $58(90.6 \%)$ & \\
$<4$ & $81(78.6 \%)$ & $6(9.4 \%)$ & \\
\hline
\end{tabular}

Bedside index for severity in acute pancreatitis BISAP, Modified CT severity index MCTSI,Hyperlipidemic acute pancreatitis HLAP 
Table4 Comparision between BISAP and MCTSI in predicting severity and local complications of HLAP

\begin{tabular}{ccccccc}
\hline Scoring system & sensitivity(\%) & specificity(\%) & PPV(\%) & NPV(\%) & AUC \\
& & & & & Youden index & \\
& & & & & & \\
\hline $\begin{array}{c}\text { MSAP and SAP } \\
\text { BISAP }\end{array}$ & 73.5 & 95.5 & 80.6 & 93.4 & 0.69 & 0.89 \\
MCTSI & 79.4 & 60.2 & 33.8 & 92.0 & 0.40 & 0.78 \\
Local complication & & & & & & \\
BISAP & 37.5 & 93.2 & 77.4 & 70.6 & 0.31 & 0.73 \\
MCTSI & 90.1 & 78.6 & 72.5 & 93.1 & 0.69 & 0.87 \\
\hline
\end{tabular}

Bedside index for severity in acute pancreatitis BISAP, Modified CT severity index MCTSI,Hyperlipidemic acute pancreatitis HLAP,Moderately severe acute pancreatitis MSAP, Severe acute pancreatitis SAP,Positive predictive value PPV,Negative predictive value NPV,Area Under the Curve AUC

Figures

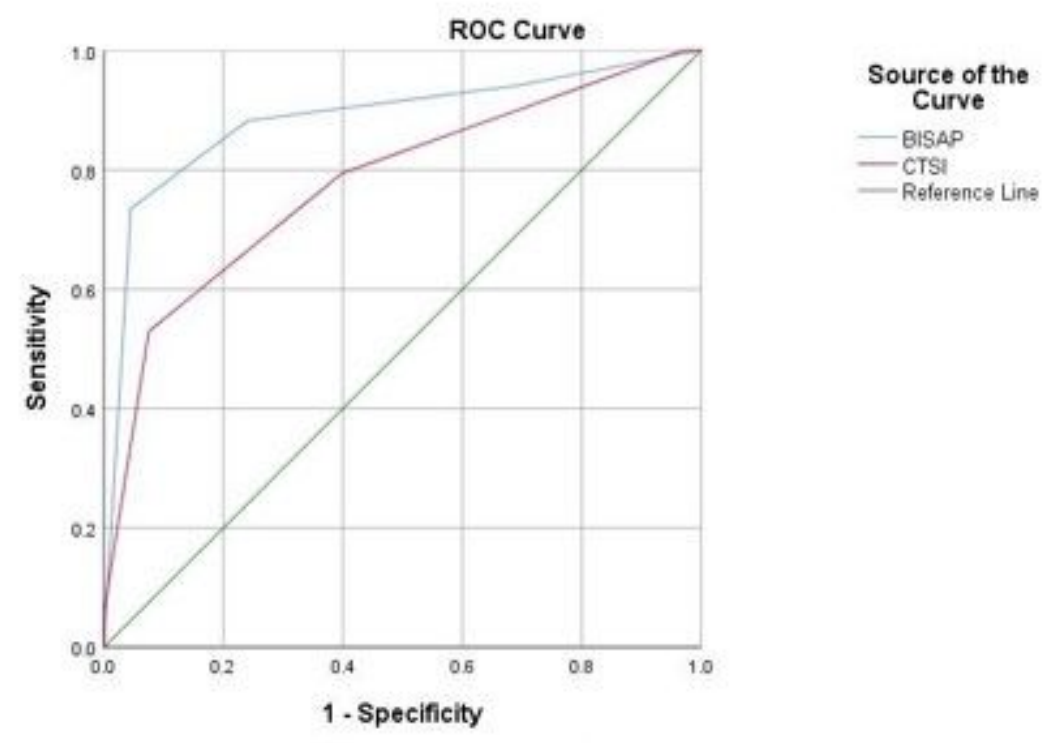


Figure 1

ROC curve of BISAP and MCTSI about the severity of HLAP

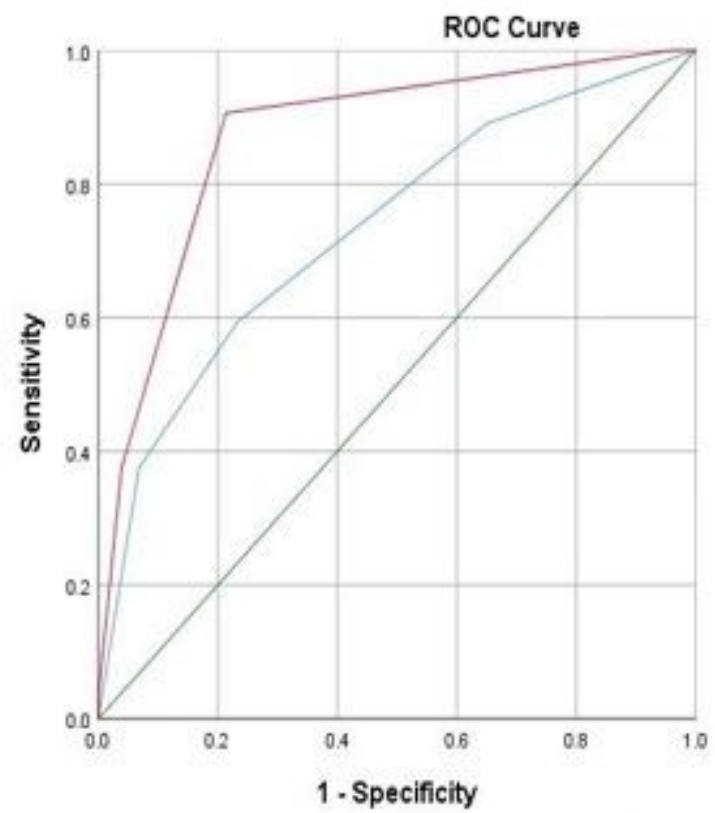

Source of the

Curve

- BISAP

- CTSI

- Reference Line

Figure 2

ROC curve of BISAP and MCTSI about local complications of HLAP

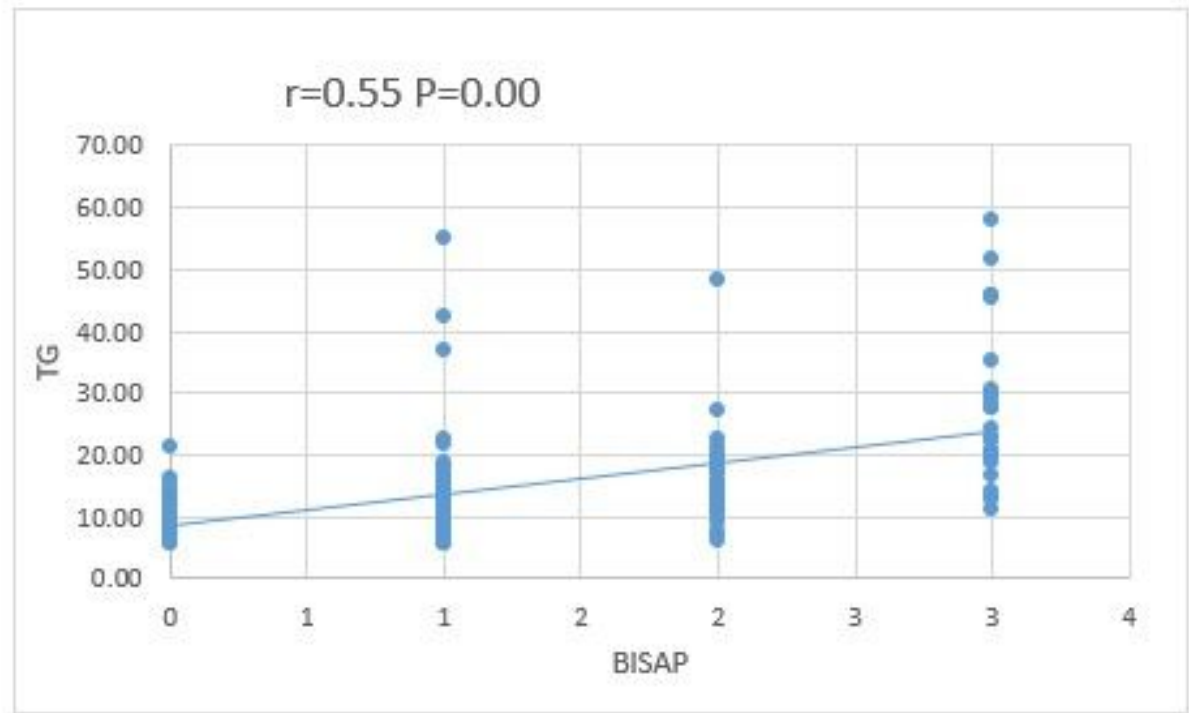


Figure 3

Correlation between BISAP and TG of HLAP 